\title{
A SURFACE POLARIZATION SURVEY OF THE MILKY WAY AND THE ZODIACAL LIGHT
}

\author{
J. STAUDE, K. WOLF, and TH. SCHMIDT \\ Max-Planck-Institut für Astronomie, Heidelberg, Germany
}

\begin{abstract}
An elliptical Surface polarimetry of the Night Sky has beeı carried out at the Boyden Observatory, South Africa. The Milky Way is linearly polarized by $p=1$ to $2 \%$ (Figure 1); 11 regions also show a circular polarized light contribution (Table I).

The amounts of circular polarization near $v \approx 0.1 \%$ or less, observed within the Zodiacal Light, are generally not exceeding twice the error of measurement: see Staude and Schmidt (1972). $(v>0$ is defined by clockwise rotation, looking against the light source).
\end{abstract}

An elliptical (i.e. linear and circular) polarization survey of the Milky Way has been undertaken, using the Heidelberg $50 \mathrm{~cm}$ Cassegrain reflector at the Boyden Observatory, South Africa, during 13 nights between 1968 May 31 and 1968 December 19. In six out of these nights the Zodiacal Light has also been observed.

The telescope was equipped with a digital on channel polarimeter and a photomultiplier EMI $6256 \mathrm{~S} / \mathrm{A}$. A circular focal diaphragm of $20^{\prime}$ was used. The observations have been carried out in two spectral regions, centered at $4200 \AA$ (during seven nights) and $5260 \AA$ (during six nights), the bandwidth being $900 \AA$ and $225 \AA$ respectively (for details see Leinert et al., 1967 and Schmidt, 1971).

Polarizing effects of the instrument including the filters, (the $5620 \AA$ one being an interference filter) were carefully checked for all Stokes' parameters during every night. The applied corrections for instrumental polarization were in the range from $0.1 \%$ to $0.4 \%$ for the relative Stokes' parameters $q=Q / I$ and $u=U / I$, and from $0.01 \%$ to $0.08 \%$ for the Stokes' parameter $v=V / I$.

The measurements of the Milky Way, covering the region $320^{\circ} \leqslant l \leqslant 40^{\circ},|b| \leqslant$ $30^{\circ}$, consisted of sequences of scans in right ascension $\alpha$ with fixed declination $\delta$, giving a reading every $4.5 \mathrm{deg}$ in $\alpha$ for integrations times of $30 \mathrm{~s}$.

The declination interval between neighbouring scans was $\Delta \delta=5^{\circ}$. By means of standard star measurements the readings of the Stokes' parameters were corrected for atmospheric extinction and converted into units $S^{10}$ (stars $10^{m} \mathrm{sq} \mathrm{deg}^{-1}$ ).

The foreground luminosity is linearly polarized because of the contributions of the Zodiacal Light and the nearby town of Bloemfontein, and it is time dependent mainly because of the air-glow fluctuations. To make a reliable separation of these foreground effects on all Stokes' parameters (between the previously mentioned sequences of scans) accurate registrations of the night sky brightness and polarization beside the Milky Way were carried out every three hours consisting of a grid of point measurements every 22.5 in declination $\delta$ and hour angle $t$, covering the whole sky.

To obtain the foreground components at the coordinates and times of the Milky Way measurements, the foreground Stokes' parameters of each night were expanded 
into spherical harmonics in the azimutal coordinate system, including terms linear and quadratic in time. With the expansion coefficients determined from the data by a least squares fit procedure, the Stokes' parameters of the foreground luminosity corresponding to the Milky Way measurements were computed and substracted from those observed in the Milky Way.

Since the surface polarization shows a much weaker color dependence then the brightness, the data obtained in the visual region were normalized to those in the blue by the averaged quotient of the intensities in the blue and visual region. Then, the Stokes' parameters of all nights were added together.

Figure 1 shows the results in general. Table I shows the 11 values of circular polarization with $|v|>2 \Delta v$ ( $\Delta v$ being the rms standard error).

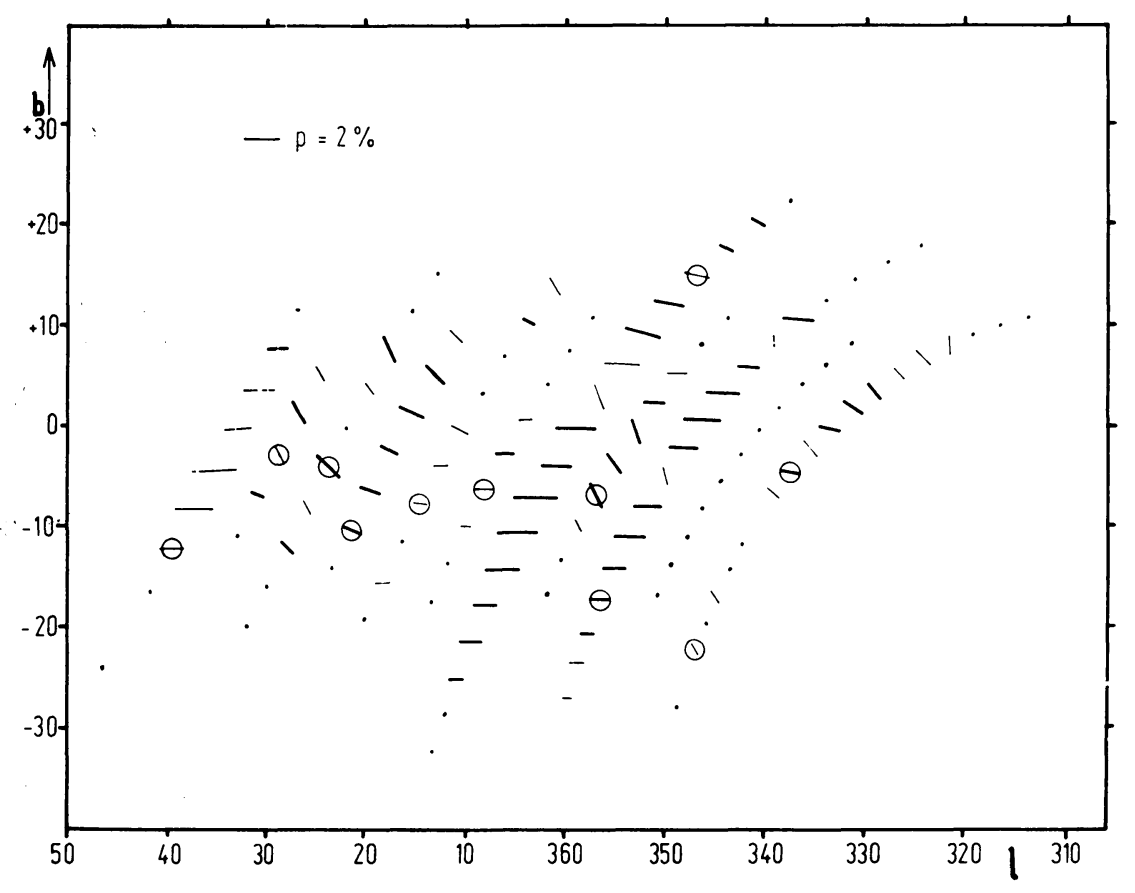

Fig. 1. Surface polarization of the Milky Way. __ $: p>2 \Delta p, \ldots$ _ $p>\Delta p, \cdot p<\Delta p$ or number of nights of observation $n<4,0: v>2 \Delta v$.

TABLE I

Circular surface polarization of the Milky Way ( $n$ : number of nights)

\begin{tabular}{rrrrrrrr}
\hline & \multicolumn{1}{c}{$b$} & \multicolumn{1}{c}{$v[\%]$} & $n$ & $l$ & $b$ & $v[\%]$ & $n$ \\
\hline $337^{\circ}$ & $-4^{\circ}$ & $0.35 \pm 0.11$ & 5 & $14^{\circ}$ & $-8^{\circ}$ & $-0.25 \pm 0.12$ & 6 \\
$347^{\circ}$ & $-22^{\circ}$ & $-0.78 \pm 0.39$ & 5 & $21^{\circ}$ & $-10^{\circ}$ & $-0.94 \pm 0.11$ & 3 \\
$356^{\circ}$ & $-17^{\circ}$ & $-0.64 \pm 0.19$ & 7 & $24^{\circ}$ & $-4^{\circ}$ & $-0.84 \pm 0.37$ & 4 \\
$357^{\circ}$ & $-7^{\circ}$ & $0.18 \pm 0.07$ & 6 & $29^{\circ}$ & $-3^{\circ}$ & $-0.72 \pm 0.07$ & 3 \\
$347^{\circ}$ & $15^{\circ}$ & $0.72 \pm 0.21$ & 5 & $40^{\circ}$ & $-12^{\circ}$ & $-0.82 \pm 0.40$ & 3 \\
$8^{\circ}$ & $-6^{\circ}$ & $0.32 \pm 0.09$ & 7 & & & & \\
\hline
\end{tabular}


The linear polarization is generally in agreement with the results of Wolstencroft (1973), but before interpreting these measurements in more detail, it is necessary to calculate whether an application of corrections is necessary, by including the reffect of scattering by atmospheric Mie particles on the surface polarization of the Milky Way.

Concerning the Zodiacal Light, the main interest was put on the parameter $V$, because many observations of the linearly polarized component have already been carried out under much better conditions.

A nonzero circular polarization component of the zodiacal light has been reported by Wolstencroft and Rose (1967), using observations at $7030 \AA$ from a sounding rocket.

The Zodiacal Light has been observed during the six nights with the visual filter. The measurements, covering the region $28^{\circ} \leqslant \varepsilon \leqslant 114^{\circ}$ and $287^{\circ} \leqslant \varepsilon \leqslant 332^{\circ},-13^{\circ}$ $\leqslant \beta \leqslant 12^{\circ}$, were arranged in the same manner as those in the Milky Way. After correction of the data for atmospheric extinction and conversion into units $S^{10}, V$-values of fields $10^{\circ} \times 10^{\circ}$ in elongation $\varepsilon$ and latitude $\beta$ were obtained averaging $n=3$ to 21 single measurements.

Altogether, the degree of circular polarization of the observed Zodiacal Light region is $|v| \leqslant 0.1 \%$. No firmly established nonzero result has been found (for details see Staude and Schmidt, 1972).

\section{Acknowledgement}

This research has been supported by the Deutsche Forschungsgemeinschaft under contract El 13/14.

\section{References}

Leinert, C., Schmidt, Th., and Schwarze, B.: 1967, Veröff. Landessternwarte Heidelberg-Königstuhl 20, 23.

Schmidt, Th.: 1971, Astron. Astrophys. 12, 456.

Staude, J. and Schmidt, Th.: 1972, Astron. Astrophys. 20, 163.

Weinberg, J. L.: 1964, Ann. Astron. 27, 718.

Wolstencroft, R. D. and Rose, L. J.: 1967, Astrophys. J. 147, 271.

Wolstencroft, R. D.: 1973, this volume, p. 99. 